\title{
Lentivirus-mediated TNF- $\alpha$ gene silencing and overexpression of osteoprotegerin inhibit titanium particle-induced inflammatory response and osteoclastogenesis in vitro
}

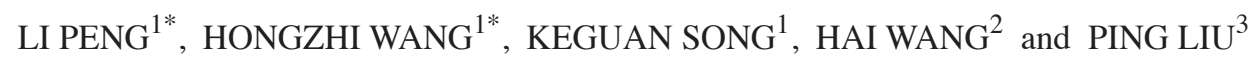 \\ ${ }^{1}$ The Third Department of Orthopaedics, The First Affiliated Hospital of Harbin Medical University, Harbin, \\ Heilongjiang 150001; ${ }^{2}$ Department of Orthopaedics, The First Affiliated Hospital of Fujian Medical University, Fuzhou, \\ Fujian 350004; ${ }^{3}$ Department of Immunology, Harbin Medical University, Harbin, Heilongjiang 150081, P.R. China
}

Received January 14, 2015; Accepted October 26, 2015

DOI: $10.3892 / \mathrm{mmr} .2015 .4620$

\begin{abstract}
Macrophages and osteoclasts release proinflammatory factors and promote osteoclastogenesis following the phagocytosis of wear particles. During this pathological process, receptor of nuclear factor $\kappa \mathrm{B}$ ligand (RANKL) and tumor necrosis factor (TNF)- $\alpha$ are critical factors contributing to resorption and the inflammatory response. The present study aimed to construct recombination lentivirus vectors carrying TNF- $\alpha$ small interfering (si)RNA and osteoprotegerin (OPG) cDNA, and to examine the effects of Lenti-siTNF $\alpha-O P G$ on the wear particle-induced inflammatory response and osteoclastogenesis in a titanium (Ti) particle-induced-inflammatory response cell model. Lenti-siTNF $\alpha-O P G$ vectors were constructed and transnfected into RAW264.7 and MC3T3-E1 cells, respectively, prior to particle stimulation. The protein levels of TNF- $\alpha$, OPG and RANKL were evaluated using western blot analysis and enzyme-linked immunosorbent assays, and the mRNA expression levels of the inflammatory factors, TNF- $\alpha$, interleukin (IL)- $1 \beta$ and IL- 6 , as well as OPG and RANKL, were measured using reverse transcription-quantitative polymerase chain reaction analysis. The activity of alkaline phosphatase (ALP) was examined using an ALP kit. In the presence of the Lenti-siTNFo-OPG vector, the mRNA expression levels of the inflammatory factors and RANKL were downregulated, as were the protein levels of TNF- $\alpha$. The mRNA expression and protein levels of OPG were upregulated, and ALP activity was increased. These
\end{abstract}

Correspondence to: Professor Keguan Song, The Third Department Of Orthopaedics, The First Affiliated Hospital of Harbin Medical University, 23 Youzheng Street, Nangang, Harbin, Heilongjiang 150001, P.R. China

E-mail: songkeguan@sohhu.com

${ }^{*}$ Contributed equally

Key words: tumor necrosis factor- $\alpha$, osteoprotegerin, lentivirus, wear particle, osteoclastogenesis findings suggested that Lenti-siTNF $\alpha-O P G$ transfection inhibited the wear particle-induced inflammatory response and osteoclastogenesis, which warrants further investigation for the prevention and/or treatment of wear particle-induced osteolysis.

\section{Introduction}

Total hip replacement improves quality of life, in terms of its reduction in pain and improved function (1). However, periprosthetic osteolysis is a major complication following total hip replacement $(2,3)$. Histopathological studies have shown the infiltration of macrophages, osteoblasts, osteoclasts and fibroblasts into peri-prosthetic tissues (4) and the interstitial membrane $(5,6)$. Each of these cells are involved in the web of interactions, which govern periprosthetic bone loss (1).

The receptor of nuclear factor $\kappa \mathrm{B}(\mathrm{RANK}) / \mathrm{RANK}$ ligand (RANKL)/osteoprotegerin (OPG) axis is at the core of the biological response of osteolysis (7), and RANKL activates nuclear factor $(\mathrm{NF}) \kappa \mathrm{B}$, which results in osteoclastgenesis. The RANKL/OPG ratio is a crucial indicator of bone mass and skeletal integrity (8). OPG is a decoy receptor for RANKL; it is secreted by osteoblasts and regulates osteoclast activity by providing an alternative binding site for RANKL, thus inhibiting the interaction between RANKL and RANK (9).

TNF- $\alpha$ is involved in the development of the osteolytic response (10) and controls the release of other proinflammatory factors, including interleukin (IL)-1 $\beta$ and IL-6 $(11,12)$. It is also reported that TNF- $\alpha$ inhibits osteoblast differentiation and promotes osteoblast apoptosis $(13,14)$. In addition, it has been suggested that TNF- $\alpha$ may act dependently (15) or independently of RANKL (10) to induce osteolysis.

TNF- $\alpha$ small interfering (si)RNA has been demonstrated to be effective in inhibiting wear particle-induced osteolysis, however certain evidence had shown that the most sensitive osteolytic response of bone to TNF- $\alpha$ is through the activation of existing osteoclasts (12). Therefore, osteoclast precursors may retain the ability to differentiate into osteoclasts through interaction with RANKL, whose decoy is OPG. OPG protein has been confirmed to have the ability to prevent periprosthetic osteolysis, however, due to the short-half life of the biological 
agent and the chronic nature of the particle-associated periprosthetic osteolysis, it is difficult to utilize conventional therapeutic methods to administer a sufficient quantity of OPG protein to osteolytic sites around the loosening prosthesis (8).

In previous years, several studies have shown that gene therapy offers a more efficient, localized, long-term option (16), compared with drugs, and have the ability prevent or treat periprosthetic osteolysis. Therefore, the present study aimed to construct a lentivirus-mediated siRNA targeting TNF- $\alpha$ in RAW264.7 cells and, at the same time, induce the overexpression of OPG in MC3T3-E1 cells, to determine whether the recombined lentivirus, Lenti-siTNF $\alpha-O P G$, has the ability to inhibit titanium (Ti) particle-induced osteolysis.

\section{Materials and methods}

Particle preparation. Commercial pure Ti particles (diameter range, $1-10 \mu \mathrm{m}$ ) with a purity of $93 \%$ were obtained from Johnson Matthey Pharma Services (Ward Hill, MA, USA). The particles were incubated in $75 \%$ ethanol for $48 \mathrm{~h}$ for sterilization and to remove endotoxin, which was followed by washing three times in sterile phosphate-buffered saline (PBS; Wuhan Boster Biological Technology, Ltd., Wuhan, China). The levels of endotoxin in the particle solutions was measured using a Limulus Amebocyte Lysate assay (Xiamen Houshiji, Ltd., Xiamen, China), and the results showed that the endotoxin level was $<0.2 \mathrm{EU} / \mathrm{ml}$. The particles were then suspended in sterile PBS at $0.1 \mathrm{mg} / \mathrm{ml}$ and stored at $4^{\circ} \mathrm{C}$ until use.

Lentiviral vector construction and recombinant lentivirus production. The siRNA target sequences (CCCAAAGGGATGAGAAGTT) were designed and cloned into a GV118 lentivirus vector (GeneChem Co., Ltd., Shanghai, China) by restriction endonuclease $\mathrm{HpaI}$ and XhoI double digestion (GeneChem Co., Ltd.) and T4 DNA ligase ligation, to construct a pLenti-PU6-siTNF- $\alpha$-PU6iEGFP backbone. Based on the mouse OPG gene sequences, PCR primers were designed (GeneChem Co., Ltd.) to clone the full length of OPG cDNA, which were cloned into the pLenti-PU6-siTNF- $\alpha$-PU6iEGFP backbone using AgeI and BamHI sites to construct the Lenti-siTNF $\alpha$-OPG vector. Following construction, the recombined lentivirus vector and pPACK Packaging Plasmid mix (Invitrogen; Thermo Fisher Scientific, Inc., Waltham, MA, USA) were cotransfected into 293T cells (GeneChem Co., Ltd.). Three short hairpin RNAs (shRNAs) were selected based on the sequences of the mouse TNF- $\alpha$ gene (GenBank: NM_013693; listed in Table I), and a scrambled shRNA served as a negative control. Preliminary experiments indicated that shRNA2 (sense, 5'-CCAACGGCATGGATCTCAA-3') downregulated TNF- $\alpha$ mRNA more markedly than the other tested shRNAs (data not shown). The pGag/Pol, pRev, pVSV-G, and recombinant lentivirus were packaged into plasmid vectors, and the recombinant lentivirus was amplified by transforming $293 \mathrm{~T}$ cells with the packaging plasmids using Lipofectamine ${ }^{\mathrm{TM}} 2000$ (Invitrogen; Thermo Fisher Scientific, Inc.). pLenti-PU6-siTNF- $\alpha$-PU6iEGFP was harvested after $48 \mathrm{~h}$. Full-length OPG cDNA was cloned into the pLenti-PU6-siTNF- $\alpha$-PU6iEGFP using AgeI and BamHI sites to construct Lenti-siTNF $\alpha$-OPG. The plasmids were then amplified by transfection into $293 \mathrm{~T}$ cells and purified with three rounds of density gradient centrifugation with $\mathrm{CsCl}$. At $48 \mathrm{~h}$ post-transfection, the lentiviruses were harvested and centrifuged at $4,000 \mathrm{x} \mathrm{g}$ for $10 \mathrm{~min}$ at $4^{\circ} \mathrm{C}$ to remove cell debris. Condensation was performed by filtration of the supernatant into a filtrate collection tube through a filter cup, followed by centrifugation at 4,000 x g for $13 \mathrm{~min}$. The filter cup was removed and a sample collection cup was inserted into the filtrate collection tube, this was then centrifuged at $1,000 \mathrm{x} g$ for $2 \mathrm{~min}$. Ultimately, a concentrated lentivirus solution was obtained, with a final titer of $1.5 \times 10^{9} \mathrm{TU} / 1$.

Cell culture. RAW264.7 mouse macrophage/monocyte cell line (American Type Culture Collection, Manassas, VA, USA) was cultured in $\alpha$-minimum essential medium ( $\alpha$-MEM; Hyclone; GE Healthcare Life Sciences, Logan, UT, USA) containing $10 \%$ fetal bovine serum (FBS; Hyclone; GE Healthcare Life Sciences), $100 \mathrm{U} / \mathrm{ml}$ penicillin (Gibco; Thermo Fisher Scientific, Inc.) and $100 \mathrm{~g} / \mathrm{ml}$ streptomycin (Gibco; Thermo Fisher Scientific, Inc.) at $37.6^{\circ} \mathrm{C}$ under $5 \%$ $\mathrm{CO}_{2}$ and $95 \%$ humidity. MC3T3-E1 (American Type Culture Collection) murine osteoblast-like cells were maintained in the same media and conditions.

3-(4,5-dimethylthiazol-2-yl)-2,5-diphenyltetrazolium bromide (MTT) assay. The cytotoxicity of the RAW264.7 and MC3T3-E1 cells transfected with Lenti-siTNF $\alpha-O P G$ was examined using the MTT assay. The cells $\left(5 \times 10^{3}\right.$ cells/well $)$ were cultured in 96-well tissue culture plates for $24 \mathrm{~h}$ and incubated with $0.5 \mathrm{mg} / \mathrm{ml} \mathrm{MTT}$ at $37^{\circ} \mathrm{C}$ for $4 \mathrm{~h}$. Following the removal of the supernatant, the insoluble formazan crystals were dissolved in $200 \mu$ l dimethyl sulfoxide, and the absorbance was measured using a Synergy HT microtiter plate reader (BioTek Instruments, Inc., Winooski, Vermont, USA) at a wavelength of $570 \mathrm{~nm}$.

Collection of conditioned media (CM). The RAW264.7 cells were plated in 24 -well plates at a density of $1.0 \times 10^{5}$ cells in complete $\alpha$-MEM. Following $24 \mathrm{~h}$ attachment at $37^{\circ} \mathrm{C}$, the cells were washed with PBS and stabilized in serum-free Dulbecco's modified Eagle's medium (Hyclone; GE Healthcare Life Sciences) for $1 \mathrm{~h}$ at $37^{\circ} \mathrm{C}$. Subsequently, the cells were subjected to Ti particles $(0.1 \mathrm{mg} / \mathrm{ml})$ with or without Lenti-siTNFa-OPG $\left(5.0 \times 10^{6} / \mathrm{ml}\right)$. Control groups were treated with equal volumes of PBS and transfection was conducted by adding $5.0 \times 10^{6} / \mathrm{ml}$ Lenti-siTNF-OPG to each well with $5 \mu \mathrm{g} / \mathrm{ml}$ polybrene and $5 \mu \mathrm{g} / \mathrm{ml}$ Enhanced Infection Solution for $72 \mathrm{~h}$. Multiplicity of infection (MOI) was determined by observation of the decrease in TNF- $\alpha$ expression and overexpression of OPG. Following $24 \mathrm{~h}$ of incubation at $37^{\circ} \mathrm{C}$, the cells in the control CM group (Cont CM), CM with Ti particles group (Ti CM) and CM with Ti particles and Lenti-siTNFa-OPG group (Ti-lenti CM) were collected, centrifuged at $1,000 \mathrm{x}$ g for $5 \mathrm{~min}$ to remove any cell debris and stored at $-20^{\circ} \mathrm{C}$ until use.

RNA isolation and reverse transcription-quantitative polymerase chain reaction $(R T-q P C R)$. The total RNA in the RAW264.7 and MC3T3-E1 cells following treatment was extracted in $1 \mathrm{ml}$ TRIzol reagent (Invitrogen; Thermo Fisher Scientific, Inc.) and cDNA was synthesized from the total RNA. qPCR was used to detect the mRNA expression levels of TNF- $\alpha$, OPG and RANKL. The sequences of the PCR 
Table I. Primers for reverse transcription-quantitative polymerase chain reaction analysis.

\begin{tabular}{lll}
\hline Target & Forward primer $\left(5^{\prime} \rightarrow 3^{\prime}\right)$ & Reverse primer $\left(3^{\prime} \rightarrow 5^{\prime}\right)$ \\
\hline TNF- $\alpha$ & TCCTCACCCACACCGTCAG & GCTGAGTTGGTCCCCCTTC \\
OPG & GATCCTGGACAGCTTCACAA & AAACAGCCCAGTGTCCATGC \\
RANKL & AGATTTGCAGGACTCGACTC & CCCACAATGTGTTGCAGTTC \\
IL-1 $\beta$ & TTCTCGCAGCAGCACATC & CAGCAGGTTATCATCATCATCC \\
IL-6 & TCCATCCAGTTGCCTTCTTG & TTTCTCATTTCCACGATTTCCC \\
GAPDH & TGGTGAAGGTCGGTGTGAAC & GCTCCTGGAAGATGGTGATGG
\end{tabular}

TNF- $\alpha$, tumor necrosis factor- $\alpha$; OPG, osteoprotegerin; RANKL, receptor of nuclear factor $\kappa$ B ligand, IL, interleukin; GAPDH, glyceraldehyde-3-phosphate dehydrogenase.

A

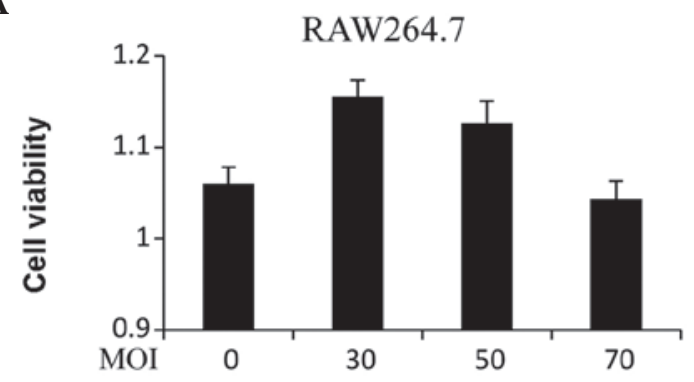

C

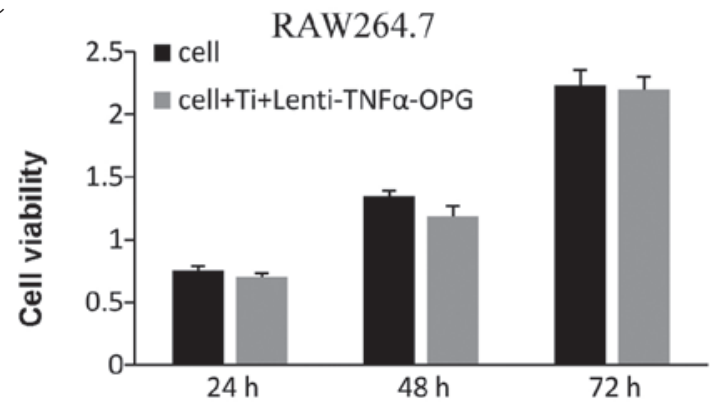

B

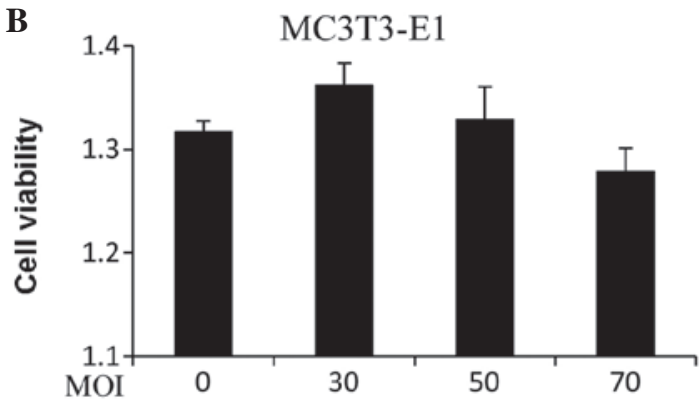

D

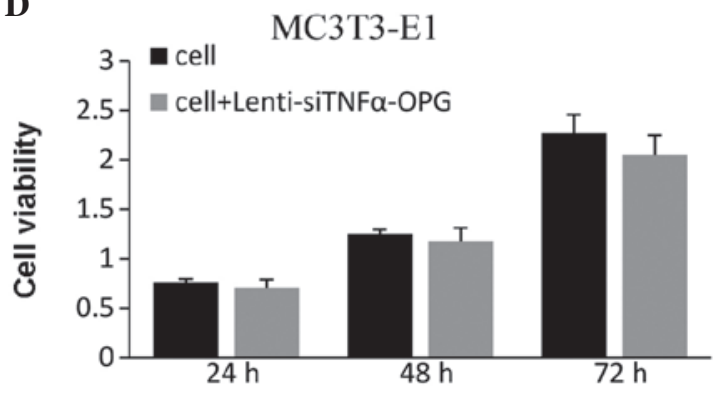

Figure 1. Effects of Lenti-siTNF $\alpha$-OPG on cell viability, determined using a 3-(4,5-dimethylthiazol-2-yl)-2,5-diphenyltetrazolium bromide assay. (A and B) RAW264.7 and MC3T3-E1 cells were transfected with different MOIs of Lenti-siTNF $\alpha$-OPG for 48 h. (C) RAW264.7 were treated with Ti and 50 MOI RAW264.7 for 24, 48 and $72 \mathrm{~h}$. (D) MC3T3-E1 cells were transfected with 50 MOI Lenti-siTNF $\alpha-O P G$ for 24, 48 and $72 \mathrm{~h}$. Data are presented as the mean \pm standard deviation. Ti, titanium; TNF- $\alpha$, tumor necrosis factor- $\alpha$; OPG, osteoprotegerin; si, small interfering; MOI, multiplicity of infection.

primers are listed in Table I. Total RNA was extracted from the MC3T3-E1 and RAW264.7 co-cultures using $1 \mathrm{ml}$ TRIzol (Invitrogen; Thermo Fisher Scientific, Inc.) according to the manufacturer's instructions. RNA purity was determined using the 260/280 $\mathrm{nm}$ absorbance ratio (NanoDrop; Thermo Fisher Scientific, Inc., Wilmington, DE, USA). First-strand cDNA was synthesized with $2 \mathrm{mg}$ total RNA (Fermentas; Thermo Fisher Scientific, Inc., Pittsburgh, PA, USA), and one-tenth of the total cDNA was used for each PCR mixture containing $5 \mu \mathrm{l}$ Express SYBR Green (Takara Bio, Inc., Otsu, Japan) and $5 \mu \mathrm{l}$ PCR Supermix (Fermentas; Thermo Fisher Scientific, Inc.). The PCR primers (0.5 $\mu 1$ upstream and downstream, respectively) used to amplify TNF- $\alpha, \mathrm{OPG}$, IL-1 $\beta$, IL-6 and glyceraldehyde-3-phosphate dehydrogenase (GAPDH) are listed in Table II. The reaction mixture $(20 \mu \mathrm{l})$ was subjected to a 45-cycle amplification in a DNA Thermal Cycler (PerkinElmer, Inc., Waltham, MA, USA) at $95^{\circ} \mathrm{C}$ for
$15 \mathrm{sec}$ and $95^{\circ} \mathrm{C}$ for $5 \mathrm{sec}$, followed by $60^{\circ} \mathrm{C}$ for $30 \mathrm{sec}$. Relative mRNA expression levels of the selected genes (TNF- $\alpha$, OPG and RANKL) were normalized to GAPDH and quantified using the $\triangle \Delta \mathrm{Cq}$ method.

Enzyme-linked immunosorbentassay(ELISA). The RAW264.7 cells were incubated with/without Ti particles in the presence or absence of Lenti-siTNFa-OPG for $24 \mathrm{~h}$, and the cell supernatants were harvested and centrifuged to remove the cell particles, as described above. Aliquots were stored at $-20^{\circ} \mathrm{C}$ for TNF- $\alpha$ measurement. A mouse TNF- $\alpha$ ELISA kit (R\&D Systems, Inc. Minneapolis, MN, USA) was used for quantitative measurement, according to the manufacturer's protocol.

Western blot analysis. The cells were lysed in radioimmunoprecipitation assay buffer (Beyotime Institute of Biotechnology, Shanghai, China) with protease inhibitors (Beyotime Institute 
A

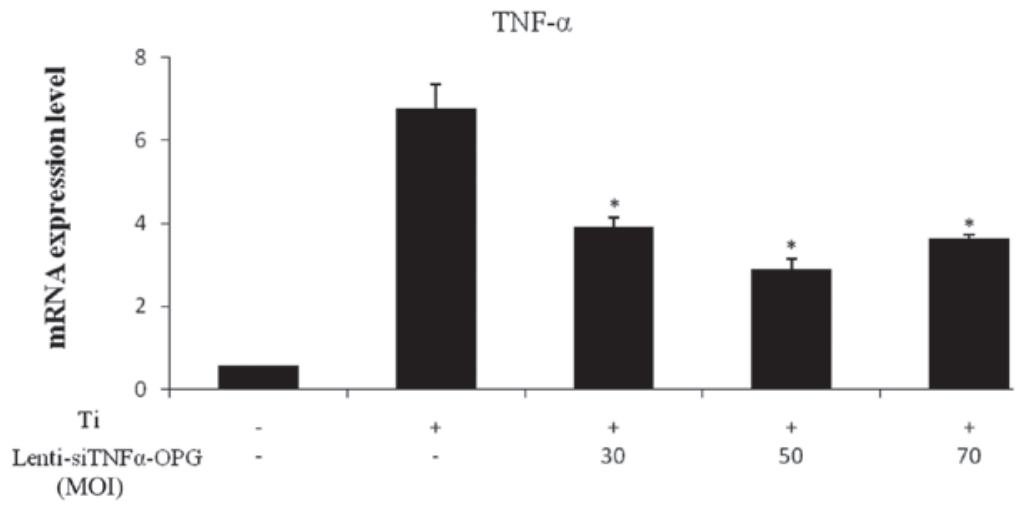

B
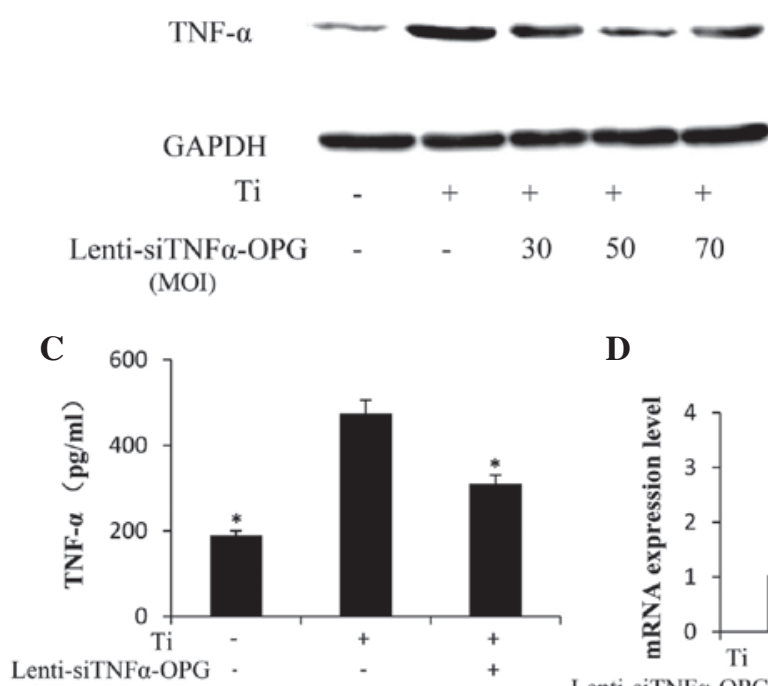

D
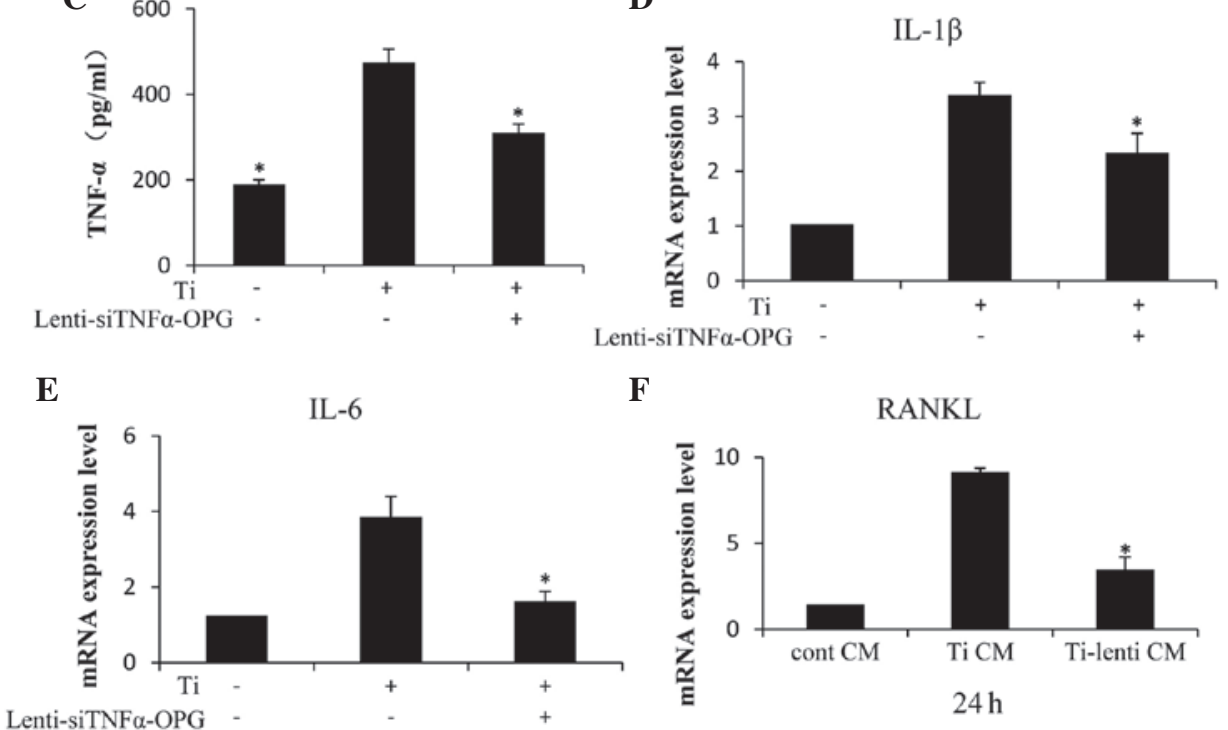

F

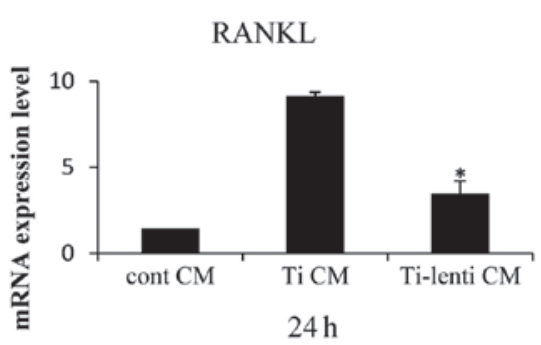

Figure 2. mRNA and protein expression levels of proinflammatory cytokines in RAW264.7 cells, and the mRNA expression of RANKL in MC3T3-E1 cells. (A) RAW264.7 cells were treated with/without Ti particles in the presence or absence of Lenti-siTNF $\alpha$-OPG for $48 \mathrm{~h}$. mRNA expression levels of TNF- $\alpha$ were significantly decreased at 50 MOI. (B) Protein levels of TNF- $\alpha$ were examined using western blotting after $48 \mathrm{~h}$. (C) Protein levels of TNF- $\alpha$ were assessed using an enzyme-linked immunosorbent assay following exposure to Ti particles, or Ti particles and 50 MOI Lenti-siTNF $\alpha$-OPG for $24 \mathrm{~h}$. (D and E) mRNA expression levels of IL-6 and IL-1 $\beta$ were significantly downregulated by Lenti-siTNF $\alpha$-OPG at 50 MOI. (F) mRNA expression levels of RANKL were significantly decreased in the MC3T3-E1 cells in the Ti-lenti CM group, compared with the Ti CM group. Data are presented as the mean \pm standard deviation. "P<0.05 vs. Ti CM. Ti, titanium; TNF- $\alpha$, tumor necrosis factor- $\alpha$; IL, interleukin; OPG, osteoprotegerin; RANKL, receptor of nuclear factor $\kappa B$ ligand; GAPDH, glyceraldehyde-3-phosphate dehydrogenase; MOI, multiplicity of infection; cont, control; CM, conditioned media; si, small interfering.

of Biotechnology). Subsequently, the protein concentrations were determined using a Bicinchoninic Acid Protein Assay kit (Beyotime Institute of Biotechnology). The total protein $(22 \mu \mathrm{g})$ was electrophoresed via 10\% SDS-polyacrylamide gel electrophoresis, transferred onto polyvinylidene difluoride membranes (PVDF; EMD Millipore, Billerica, MA, USA) and blocked in TBS for $1 \mathrm{~h}$. The PVDF membranes were incubated overnight at $4{ }^{\circ} \mathrm{C}$ with rabbit anti-mouse monoclonal TNF- $\alpha$ (1:300; Abcam, Cambridge, MA; cat. no. ab11564) and rabbit anti-mouse polyclonal OPG (1:200) antibodies
(Abcam; cat. no. ab9986). GAPDH served as a protein loading control. Following incubation with the primary antibody, the membranes were washed twice with TBST for $10 \mathrm{~min}$ and then washed with TBS for $10 \mathrm{~min}$. Subsequently, the blots were incubated with goat anti-rabbit monoclonal secondary antibody (1:500; cat. no. 7074P2; Cell Signaling Technology, Inc., Shanghai, China) at $20^{\circ} \mathrm{C}$ for $2 \mathrm{~h}$. Following incubation with the secondary antibody, the membranes were washed twice in TBST for $10 \mathrm{~min}$ and then washed with TBS for $10 \mathrm{~min}$. Following incubation, the proteins were detected by enhanced 

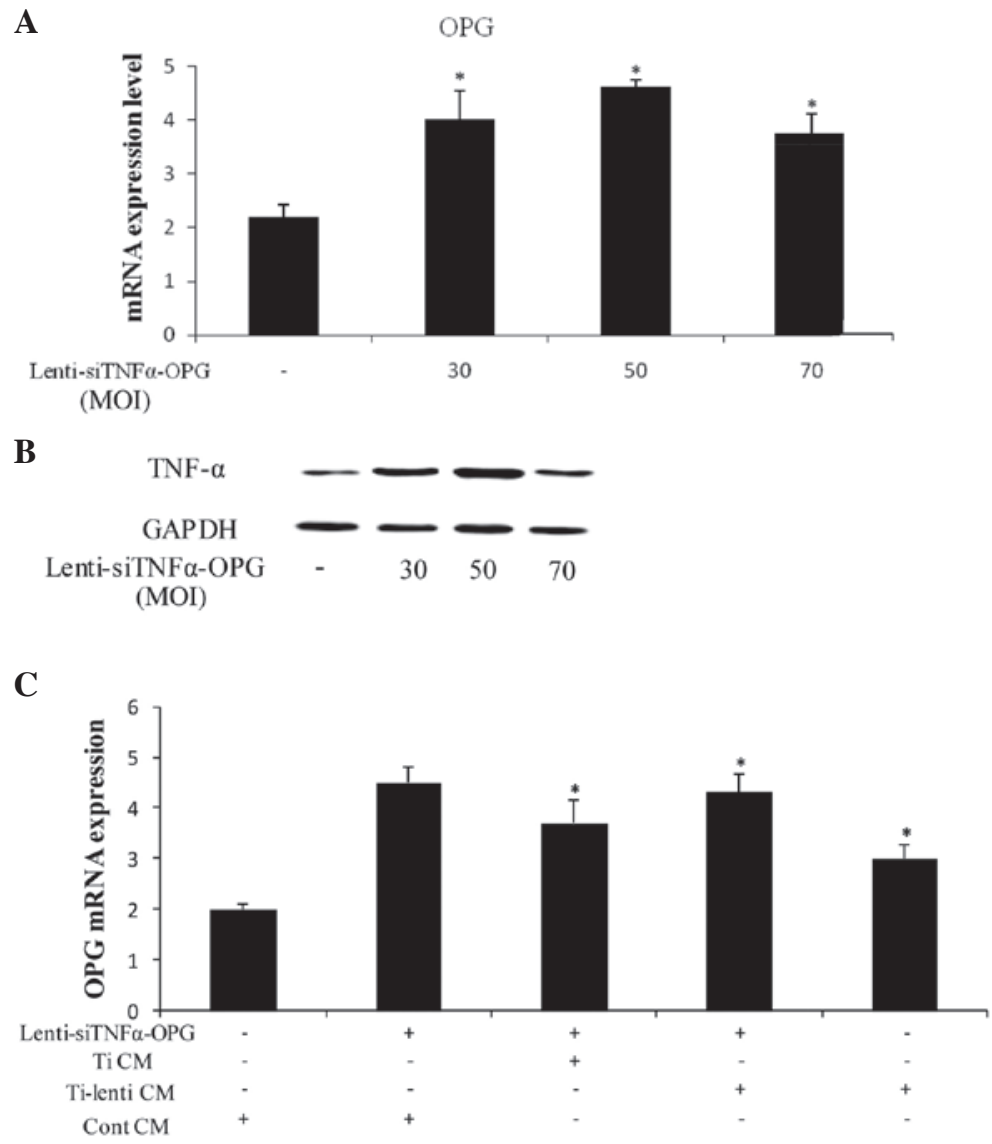

Figure 3. Expression levels of OPG in MC3T3-E1 cells treated with Lenti-siTNF $\alpha$-OPG and/or different CM. (A) RAW264.7 were transfected with Lenti-siTNFa-OPG at different MOIs for $48 \mathrm{~h}$. The mRNA expression of OPG was significantly increased at 50 MOI. "P<0.05 vs. 0 Lenti-siTNFa-OPG. (B) Protein levels of OPG were determined using western blotting after $48 \mathrm{~h}$. (C) Expression levels of OPG were significantly increased in the MC3T3-E1 cells in the Lenti-siTNFo-OPG and Ti-lenti CM-treated group, compared with the Lenti-siTNFo-OPG and Ti CM-treated and Ti-lenti-treated groups. Data are presented as the mean \pm standard deviation. ${ }^{*} \mathrm{P}<0.05$ vs. Cont $\mathrm{CM}+$ Lenti-siTNF $\alpha-\mathrm{OPG}$. Ti, titanium; TNF- $\alpha$, tumor necrosis factor- $\alpha$; IL, interleukin; OPG, osteoprotegerin; RANKL, receptor of nuclear factor $\kappa \mathrm{B}$ ligand; GAPDH, glyceraldehyde-3-phosphate dehydrogenase; MOI, multiplicity of infection; Cont, control; CM, conditioned media; si, small interfering.

chemiluminescence with BeyoECL Plus (Beyotime Institute of Biotechnology) and scanned using Quantity One analysis software, version 4.6 (Bio-Rad Laboratories, Inc., Hercules, CA, USA).

ALP activity assay. ALP activity was measured using a QuantiChrom $^{\mathrm{TM}}$ Alkaline Phosphatase Assay kit (BioAssay Systems, Hayward, CA, USA). In brief, the culture medium was removed, and following being washed with PBS, the cells were lysed in $0.5 \mathrm{ml} 0.2 \%$ Triton $\mathrm{X}-100$ in distilled water with agitation for $20 \mathrm{~min}$ at room temperature. The samples were then incubated with a mixture of assay buffer $(\mathrm{pH} 10.5)$, $5 \mathrm{mM} \mathrm{Mg}$ acetate (final) and $10 \mathrm{mM}$ pNPP liquid substrate at room temperature for $10 \mathrm{~min}$. The optical density at $405 \mathrm{~nm}$ was determined $(\mathrm{t}=0)$, and this was measured again after $4 \mathrm{~min}$ ( $\mathrm{t}=4 \mathrm{~min}$ ) on a plate reader (Multiskan Plus; Thermo Fisher Scientific, Inc.). The quantity of released nitrophenolate was calculated photometrically, according to the manufacturer's protocol.

Statistical analysis. Data from three independent experiments were analyzed and are presented as the mean \pm standard deviation. Differences between groups were analyzed using one-way analysis of variance. $\mathrm{P}<0.05$ was considered to indicate a statistically significant difference. All statistical analyses were performed using SPSS 11.0 software (SPSS, Inc., Chicago, IL, USA).

\section{Results}

Variation in the MOI of Lenti-siTNF $\alpha-O P G$ transfection has no effect on cell viability. The results of the MTT assay revealed no significant differences among the RAW264.7 and MC3T3-E1 cells transfected with different MOIs (30, 50 and $70 \mathrm{MOI}$ ) of Lenti-siTNFa-OPG for $48 \mathrm{~h}$ (Fig. 1A and B). As the mRNA expression levels of TNF- $\alpha$ and OPG were significantly downregulated at $50 \mathrm{MOI}$, the present study analyzed the viability of RAW264.7 cells treated with Ti particles and 50 MOI Lenti-siTNFa-OPG at 24, 48 and $72 \mathrm{~h}$, which showed no significant difference, compared with the untransfected cells (Fig. 1C). Similarly, no significant difference was observed between the viability of the MC3T3-E1 cells transfected with 50 MOI Lenti-siTNFa-OPG (Fig. 1D) at each time point, compared with the untransfected cells.

Lenti-siTNF $\alpha-O P G$ inhibits the expression of cytokines in RAW264.7 cells. Compared with 30 and 70 MOI, the mRNA expression of TNF- $\alpha$ in the RAW264.7 cells transfected with 

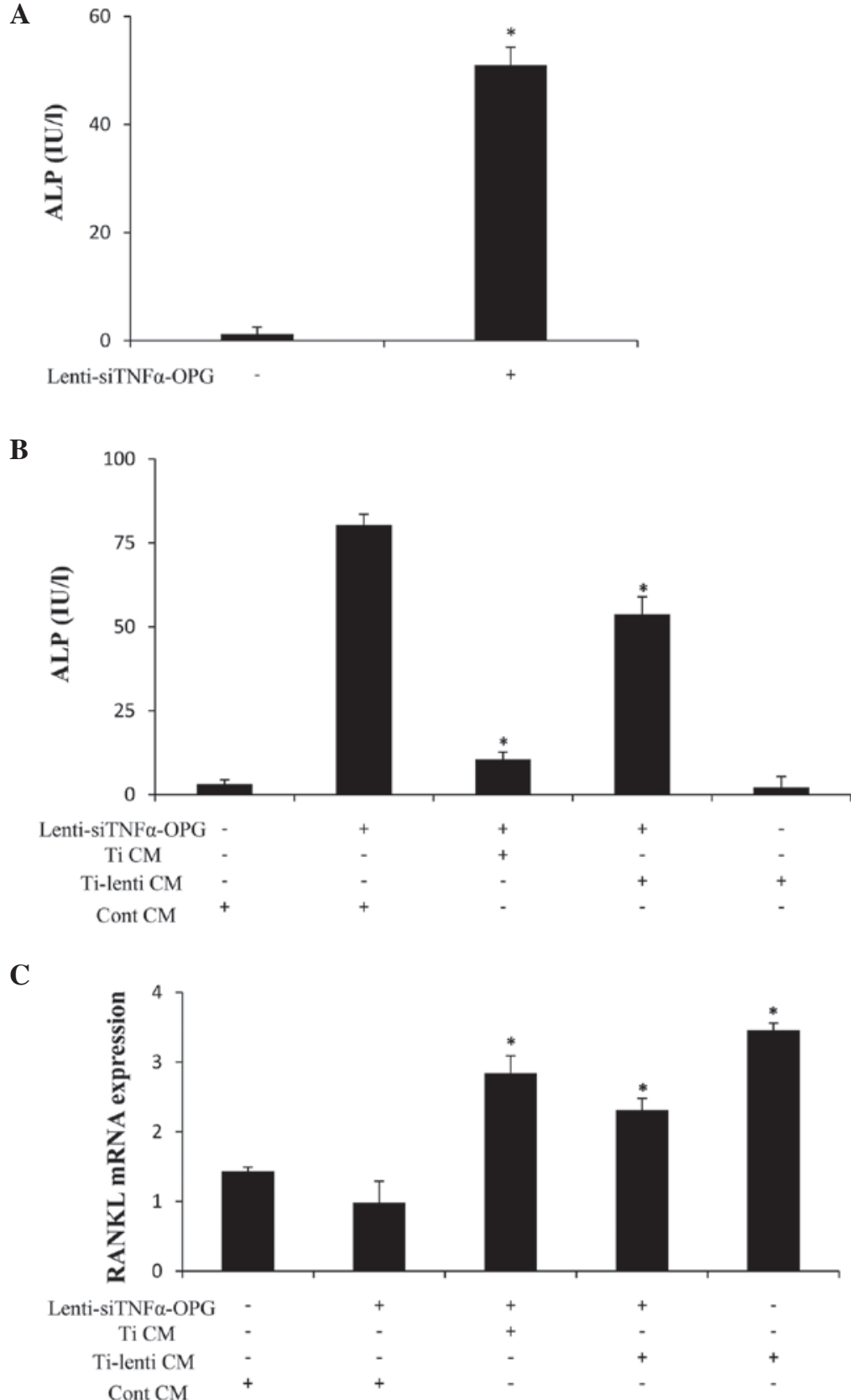

Figure 4. ALP activity and mRNA expression levels of RANKL in MC3T3-E1 cells. (A) MC3T3-E1 cells transfected with 50 MOI Lenti-siTNFa-OPG for $48 \mathrm{~h}$. ALP activity was significantly higher, compared with the control. " $\mathrm{P}<0.05$ vs. cont. (B) ALP activity was significantly increased in the MC3T3-E1 cells of the Lenti-siTNFo-OPG and Ti-lenti CM-treated group, compared with the Lenti-siTNF $\alpha$-OPG and Ti CM and Ti-lenti-treated groups. "P<0.05 vs. cont $\mathrm{CM}+$ Lenti-siTNF $\alpha$-OPG. (C) mRNA expression levels of RANKL were significantly decreased in the MC3T3-E1 cells of the Lenti-siTNF $\alpha$-OPG and Ti-lenti CM-treated group, compared with the Lenti-siTNF $\alpha$-OPG and Ti CM and Ti-lenti-treated groups. "P<0.05 vs. cont CM + Lenti-siTNF $\alpha-O P G$. Data are presented as the mean \pm standard deviation. Ti, titanium; TNF- $\alpha$, tumor necrosis factor- $\alpha$; IL, interleukin; OPG, osteoprotegerin; RANKL, receptor of nuclear factor кB ligand; GAPDH, glyceraldehyde-3-phosphate dehydrogenase; MOI, multiplicity of infection; cont, control; CM, conditioned media; si, small interfering.

50 MOI Lenti-siTNF $\alpha$-OPG was lowest at $48 \mathrm{~h}$ (Fig. 2A). The protein expression of TNF- $\alpha$, determined using western blot analysis, revealed similar results (Fig. 2B). These results confirmed that 50 MOI Lenti-siTNF $\alpha$-OPG significantly reduced the expression levels of TNF- $\alpha$ in the RAW264.7 cells treated with $0.1 \mathrm{mg} / \mathrm{ml}$ Ti particles. Following $24 \mathrm{~h}$ incubation with a combination of Ti particles and $50 \mathrm{MOI}$ Lenti-siTNF $\alpha$-OPG, the protein expression of TNF- $\alpha$ was inhibited in the RAW264.7 cells, compared with the with cells in the $\mathrm{Ti}$ CM group, as determined using ELISA analysis (Fig. 2C). To evaluate the particle-induced inflammatory response, the present study examined the expression levels of proinflammatory cytokines, including IL-1 $\beta$ and IL-6. It was found that the downregulation of TNF $\alpha$ by siTNF $\alpha$ resulted in decreases in the mRNA expression levels of IL-1 $\beta$ (Fig. 2D) and IL-6 (Fig. 2E), compared with the Ti CM group, which indicated that TNF- $\alpha$ may control the mRNA expression levels of IL- 6 and IL- $1 \beta$ mRNA. It was also observed that the mRNA expression of RANKL in the MC3T3-E1 cells decreased markedly when cultured 
in lenti-Ti CM, compared with the Ti CM group, which indicated that TNF- $\alpha$ may have an effect on the expression of RANKL (Fig. 2F).

Lenti-siTNF $\alpha-O P G$ upregulates the expression of OPG in MC3T3-E1 cells. To assess the mRNA expression levels of OPG, the MC3T3-E1 cells treated with different MOIs $(30,50$ and 70) were examined using RT-qPCR. The results showed that the expression level of OPG was highest at an MOI of 50 at $48 \mathrm{~h}$ (Fig. 3A). At $48 \mathrm{~h}$ post-transfection with the different MOIs of Lenti-siTNF $\alpha$-OPG, overexpression of OPG protein was demonstrated in the MC3T3-E1 cells using western blot analysis (Fig. 3B). As expected, the MC3T3-E1 cells transfected with 50 MOI Lenti-siTNF $\alpha$-OPG exhibited higher protein expression levels of OPG, compared with those transfected with 30 and 70 MOI. The present study also examined the protein expression levels of OPG in the OPG-overexpressing MC3T3-E1 cells culture in Cont CM, Ti CM and Ti-lenti CM using ELISA. The Lenti-siTNF $\alpha$-OPG and Ti-lenti CM-treated group exhibited the highest protein expression of OPG, compared with the Ti CM and Ti-lenti CM-treated groups (Fig. 3C), suggesting that the overexpression of OPG is more marked when the expression of TNF- $\alpha$ is decreased.

Lenti-siTNF $\alpha-O P G$ promotes osteoblast differentiation and inhibits osteoclastogenesis in transfected MC3T3-E1 cells. ALP is a marker of matrix maturation and, during differentiation from mesenchymal cells to mature osteoblasts, ALP begins to be expressed in osteoprogenitors and is expressed at high levels in mature osteoblasts (17). Using an ALP kit, the present study assessed the activity of ALP in the MC3T3-E1 cells transfected with or without Lenti-siTNF $\alpha-O P G$ for $48 \mathrm{~h}$. The results revealed that ALP activity in the MC3T3-E1 cells increased following transfection with Lenti-siTNF $\alpha$-OPG (Fig. 4A). It was also found that ALP activity was significantly higher in the OPG-overexpressing MC3T3-E1 cells when treated with the different CM (Fig. 4B). These data indicated that Lenti-siTNF $\alpha$-OPG transfection alleviated Ti particle-induced osteolysis by promoting osteoblast differentiation.

RANKL has a high level of involvement in osteoclastogenesis due to its binding to the receptor activator of RANK. In the present study, RT-qPCR analysis revealed differences in the mRNA expression levels of RANKL in the OPG-overexpressing MC3T3-E1 cells cultured in Cont CM, Ti CM and Ti-lenti CM (Fig. 4C). The Lenti-siTNFa-OPG and Ti-lenti CM-treated group revealed the lowest mRNA expression level of RANKL, compared with the Ti CM and Ti-lenti CM-treated groups. These results indicated that the Lenti-siTNF $\alpha$-OPG may have suppressed Ti particle-induced osteoclastogenesis.

\section{Discussion}

At present, there is no satisfactory treatment option for periprosthetic osteolysis, with the exception of revision, which requires complicated and expensive surgery, and is frequently associated with considerable patient morbidity, and even mortality rates (18). There has been a focus on nonsurgical methods to prevent aseptic loosening (19), however, there remains no satisfactory method for the prevention of aseptic loosening of joint protheses. To the best of our knowledge, the present study is the first study to address the effects of the combination of TNF- $\alpha$ siRNA and overexpression of OPG by construction of a recombined lentivirus.

RANKL is essential for the promotion of osteoclastogenesis. It binds to its signaling receptor, RANK, on the membranes of macrophages and osteoclast precursors, thereby providing signals required for their survival, maturation and activation (20). Mature osteoclasts secret large quantities of inflammatory factors, including TNF- $\alpha$, IL- $1 \beta$ and IL-6, which in turn activate osteoclasts and prompt an inflammatory response (10). Among these inflammatory factors, TNF- $\alpha$ acts as a link between inflammatory processes and osteoclastogenesis. It is involved in the osteolytic response predominantly by two mechanisms: An indirect mechanism, in which TNF- $\alpha$ enhances the inflammatory response by promoting the expression levels of RANKL, IL-6 and IL-1 $\beta$ (21) and a direct mechanism, in which TNF- $\alpha$ synergizes with RANKL to enhance osteoclast formation of bone erosions (22). In the present study, Lenti-siTNF $\alpha$-OPG transfection inhibited osteolysis by the two mechanisms. It decreased the level of TNF- $\alpha$ secreted by osteoclasts and subsequently suppressed the expression levels of IL-1 $\beta$ and IL- 6 stimulated by the Ti particles. It also decreased the expression of TNF- $\alpha$, which ma have led to the reduction in the expression of RANKL, controlling osteoclast maturation and function (23). As shown in the data of the present study, Lenti-siTNF $\alpha$-OPG transfection effectively reduced the expression of RANKL in the MC3T3-E1 cells by interfering with the levels of the proinflammatory cytokines secreted from the RAW264.7 cells induced by Ti particles. In addition, TNF- $\alpha$ and RANKL support osteoclast survival, therefore, downregulation in the expression of TNF- $\alpha$ reduces the numbers of osteoclasts (24). Therefore, Lenti-siTNF $\alpha$-OPG transfection may prevent the inflammatory response, which is important in periprosthetic osteolysis.

ALP is a vital early marker of matrix maturation, which is expressed in preosteoblasts during osteoblast differentiation and exhibits upregulated expression levels in mature osteoblasts and downregulated expression levels in osteocytes (25). In the present study, Lenti-siTNFo-OPG transfection promoted osteoblast differentiation, accompanied by the expression of ALP. Furthermore, the transfection of Lenti-siTNFo-OPG resulted in suppression of the expression of RANKL and the inhibition of osteoclastogenesis (26). Compared with preosteoblasts, the ratio of RANKL to OPG is markedly higher in mature osteoblasts (17). In addition, in the presence of TNF- $\alpha$, the level of apoptosis in mature osteoblasts is higher than in preosteoblasts (27). Therefore, Lenti-siTNFa-OPG transfection may induce osteoblast maturation and inhibit osteoclast differentiation.

OPG has been identified as a negative regulator of the RANKL/RANK/OPG axis (28). Overexpression of OPG upregulates the OPG/RANKL ratio, inhibiting the interaction of RANKL and RANK, leading to suppression of the inflammatory response and osteoclastogenesis. The results of the present study clearly revealed that Lenti-siTNF $\alpha$-OPG transfection increase the expression of OPG in the MC3T3-E1 cells. In addition, comparison of the mRNA expression levels of RANKL 
among the MC3T3-E1 cells of the Lenti-siTNF $\alpha-O P G$ and Ti-lenti CM-treated, Lenti-siTNF $\alpha-O P G$ and Ti CM-treated and Ti-lenti CM-treated groups, the Lenti-siTNF $\alpha-O P G$ and Ti-lenti CM-treated MC3T3-E1 cells exhibited the lowest mRNA expression level of RANKL. This indicated that the combination of the decreased expression of TNF- $\alpha$ in RAW264.7 cells with increased the expression of OPG in MC3T3-E1 cells downregulated the mRNA expression levels of RANKL more effectively. As RANKL is critical for osteoclastogenesis, it was hypothesized that Lenti-siTNF $\alpha-O P G$ transfection may be more effective in inhibiting osteoclastogenesis, compared with siRNA that target only the downregulation of TNF- $\alpha$ or upregulation of OPG.

Gene therapy is an attractive option for treatment of osteolysis. Major problems in the approaches to treat localized chronic inflammatory/osteolytic disorders, including aseptic loosening, include the lack of adequate suppressive agents and effective specific therapeutic delivery systems (18). Although the conventional system of administering biological drugs, including bisphosphonates, relies on vascular perfusion to the local sites of loosening, viral vector mediated gene therapy provides a novel means of delivering therapeutic genes to the site of disease to express gene products in a persistent and localized manner (18). In addition, investigations have been performed on TNF- $\alpha$ siRNA or OPG cDNA gene delivery $(29,30)$, and they have been demonstrated to be more potent, efficacious and cost-effective in inhibiting wear particle-induced inflammatory response and osteoclastogenesis. As a promising vector of siRNA, the lentivirus compares favorably with other transgenic methods for transducing genes in vivo. Transfection with a lentivirus is more efficient than other methods due to its stable expression of siRNA in mammalian cells (31). Overall, Lenti-siTNF $\alpha-O P G$ appears to be an effective mechanism to prevent Ti particle-induced osteolysis.

For investigations aim to examine the effect of Lenti-siTNF $\alpha-O P G$ on periprosthetic osteolysis in vitro, and to fully understand the molecular mechanisms underlying the therapeutic effects, as well as safety concerns.

In conclusion, the present study demonstrated that the inhibition of TNF- $\alpha$ and overexpression of OPG by recombined lentivirus transfection effectively alleviated the $\mathrm{Ti}$ particle-induced inflammatory response and osteoclastogenesis in vitro, and indicated that Lenti-siTNF $\alpha-O P G$ may be a potential therapeutic method for the prevention of $\mathrm{Ti}$ particle-induced osteolysis.

\section{Acknowledgements}

The study was supported by funding from the National Natural Science Foundation of China (grant no. 81170386). All experiments were performed in the Laboratory of Cardiology of the First Affiliated Hospital of Harbin Medical University (Harbin, China). The authors would like to thank the managers and staff for their hospitability, time and opinions.

\section{References}

1. Ollivere B, Wimhurst JA, Clark IM and Donell ST: Current concepts in osteolysis. J Bone Joint Surg Br 94: 10-15, 2012.
2. Gallo J, Kamínek P, Tichá V, Riháková P and Ditmar R: Particle disease. A comprehensive theory of periprosthetic osteolysis: A review. Biomed Pap Med Fac Univ Palacky Olomouc Czech Repub 146: 21-28, 2002.

3. Harris WH: Wear and periprosthetic osteolysis: The problem. Clin Orthop Relat Res: 66-70, 2001.

4. Schmalzried TP, Jasty M and Harris WH: Periprosthetic bone loss in total hip arthroplasty. Polyethylene wear debris and the concept of the effective joint space. J Bone Joint Surg Am 74: 849-863, 1992.

5. Agarwal S: Osteolysis-basic science, incidence and diagnosis. Curr Orthopaed 18: 220-231, 2004

6. Purdue PE, Koulouvaris P, Nestor BJ and Sculco TP: The central role of wear debris in periprosthetic osteolysis. HSS J 2: 102-113, 2006.

7. Abu-Amer Y, Darwech I and Clohisy JC: Aseptic loosening of total joint replacements: Mechanisms underlying osteolysis and potential therapies. Arthritis Res Ther 9 (Suppl 1): S6, 2007.

8. Boyce BF and Xing L: Biology of RANK, RANKL and osteoprotegerin. Arthritis Res Ther 9 (Suppl 1): S1, 2007.

9. Takahashi N, Maeda K, Ishihara A, Uehara S and Kobayashi Y. Regulatory mechanism of osteoclastogenesis by RANKL and Wnt signals. Front Biosci (Landmark Ed) 16: 21-30, 2011.

10. Kwan Tat S, Padrines M, Théoleyre S, Heymann D and Fortun Y: IL-6, RANKL, TNF-alpha/IL-1: Interrelations in bone resorption pathophysiology. Cytokine Growth Factor Rev 15: 49-60, 2004.

11. Akisue T, Bauer TW, Farver CF and Mochida Y: The effect of particle wear debris on NFkappaB activation and pro-inflammatory cytokine release in differentiated THP-1 cells. J Biomed Mater Res 59: 507-515, 2002.

12. Greenfield EM and Bechtold J; Implant Wear Symposium 2007 Biologic Work Group: What other biologic and mechanical factors might contribute to osteolysis? J Am Acad Orthop Sur 16 (Suppl): S56-S62, 2008

13. Abbas S, Zhang YH, Clohisy JC and Abu-Amer Y: Tumor necrosis factor-alpha inhibits pre-osteoblast differentiation through its type-1 receptor. Cytokine 22: 33-41, 2003.

14. Xu J, Wu HF, Ang ES, Yip K, Woloszyn M, Zheng MH and Tan RX: NF-kappaB modulators in osteolytic bone diseases. Cytokine Growth Factor Rev 20: 7-17, 2009.

15. Yoshino T, Yamaguchi M, Shimizu M, Yamada K and Kasai K: TNF- $\alpha$ aggravates the progression of orthodontically-induced inflammatory root resorption in the presence of RANKL. J Hard Tissue Biol 23: 155-162, 2014.

16. Froelich S, Tai A and Wang P: Lentiviral vectors for immune cells targeting. Immunopharmacol Immunotoxicol 32: 208-218, 2010.

17. Rucci N, Rufo A, Alamanou M and Teti A: Modeled microgravity stimulates osteoclastogenesis and bone resorption by increasing osteoblast RANKL/OPG ratio. J Cell Biochem 100: 464-473, 2007.

18. Philpott A, Weston-Simons JS, Grammatopoulos G, Bejon P, Gill HS, McLardy-Smith P, Gundle R, Murray DW and Pandit H: Predictive outcomes of revision total hip replacement-A consecutive series of 1176 patients with a minimum 10-year follow-up. Maturitas 77: 185-190, 2014.

19. Goodman SB, Trindade M, Ma T, Genovese M and Smith RL: Pharmacologic modulation of periprosthetic osteolysis. Clin Orthop Relat Res: 39-45, 2005.

20. Wang CT, Lin YT, Chiang BL, Lee SS and Hou SM: Over-expression of receptor activator of nuclear factor-kappaB ligand (RANKL), inflammatory cytokines and chemokines in periprosthetic osteolysis of loosened total hip arthroplasty. Biomaterials 31: 77-82, 2010.

21. Teitelbaum SL: Osteoclasts; culprits in inflammatory osteolysis. Arthritis Res Ther 8: 201, 2006.

22. Ritchlin CT, Haas-Smith SA, Li P, Hicks DG and Schwarz EM: Mechanisms of TNF-alpha and RANKL mediated osteoclastogenesis and bone resorption in psoriatic arthritis. J Clin Invest 111: 821-831, 2003.

23. He X, Andersson G, Lindgren U and Li Y: Resveratrol prevents RANKL-induced osteoclast differentiation of murine osteoclast progenitor RAW264.7 cells through inhibition of ROS production. Biochem Biophys Res Commun 401: 356-362, 2010.

24. Adapala NS, Barbe MF, Langdon WY, Nakamura MC, Tsygankov AY and Sanjay A: The loss of Cbl-phosphatidylinositol 3-kinase interaction perturbs RANKL-mediated signaling, inhibiting bone resorption and promoting osteoclast survival. J Biol Chem 285: 36745-36758, 2010.

25. Inose H, Ochi H, Kimura A, Fujita K, Xu R, Sato S, Iwasaki M, Sunamura S, Takeuchi Y, Fukumoto S, et al: A microRNA regulatory mechanism of osteoblast differentiation. Proc Natl Acad Sci USA 106: 20794-20799, 2009. 
26. Bonnelye E, Chabadel A, Saltel F and Jurdic P: Dual effect of strontium ranelate: Stimulation of osteoblast differentiation and inhibition of osteoclast formation and resorption in vitro. Bone 42: 129-138, 2008.

27. Almeida M, Han L, Ambrogini E, Weinstein RS and Manolagas SC: Glucocorticoids and tumor necrosis factor $\alpha$ increase oxidative stress and suppress Wnt protein signaling in osteoblasts. J Biol Chem 286: 44326-44335, 2011.

28. Pivonka P, Zimak J, Smith DW, Gardiner BS, Dunstan CR, Sims NA, Martin TJ and Mundy GR: Theoretical investigation of the role of the RANK-RANKL-OPG system in bone remodeling. J Theor Biol 262: 306-316, 2010.
29. Dong L, Wang R, Zhu YA, Wang C, Diao H, Zhang C, Zhao J and Zhang J: Antisense oligonucleotide targeting TNF-alpha can suppress Co-Cr-Mo particle-induced osteolysis. J Orthop Res 26: 1114-1120, 2008

30. Goater JJ, O'Keefe RJ, Rosier RN, Puzas JE and Schwarz EM: Efficacy of ex vivo OPG gene therapy in preventing wear debris induced osteolysis. J Orthop Res 20: 169-173, 2002.

31. Kay MA, Glorioso JC and Naldini L: Viral vectors for gene therapy: The art of turning infectious agents into vehicles of therapeutics. Nat Med 7: 33-40, 2001. 